\title{
Transfert du radiocésium présent dans du foin contaminé par les retombées de Tchernobyl à des brebis gestantes et à leurs produits à différents âges
}

\author{
F. DABURON*, G. FAYART, M. BARADAT, S. LIBOTTE*
}

(Manuscrit reçu le 10 avril 1992)

RÉSUMÉ Quatre brebis gestantes ont reçu du foin contaminé par les retombées de l'accident de Tchernobyl, pendant environ 1 mois avant et un mois après le part. Le niveau de contamination $\left({ }^{134} \mathrm{Cs}\right.$ et $\left.{ }^{137} \mathrm{Cs}\right)$ des mères et des agneaux a été déterminé par comptage in toto ; la quantité de lait ingérée par les agneaux a été estimée par leur gain de poids journalier. Entre $\mathrm{J} 10$ et $\mathrm{J} 30$ après le part, la valeur moyenne des transferts a été de $4,7 \pm 1,4 \%$ pour le lait et $7,9 \pm 1,7 \%$ pour la viande. A la naissance, la charge corporelle moyenne des agneaux était égale à $3,2 \pm 0,8 \%$ celle de mère ; le coefficient moyen de rétention du césium ingéré était au sevrage (J30) de $39 \pm 6 \%$ pour les agneaux et de $27,3 \pm 3,2 \%$ pour les brebis. La période de décontamination spontanée était pour les agneaux après le sevrage d'environ 14,5 jours (courbe monoexponentielle) et d'environ 26 jours pour la composante longue de la courbe bi-exponentielle chez les brebis.

Les transferts à la viande, les paramètres de charge et de décontamination spontanée ont été également étudiés : 1) chez 4 agneaux de 1 à 3 mois après le sevrage, 2) chez 3 jeunes animaux âgés de 9 à 10 mois. Les valeurs des différents paramètres du transit du césium chez les nouveaux-nés et chez les jeunes animaux sont discutées en rapport avec la gestion d'un accident de contamination.

ABSTRACT Contamined hay harvested in the south-east of France in June 1986 was fed to 4 pregnant ewes from about 1 month before to 1 month after lambing. The caesium body burdens $\left({ }^{137} \mathrm{Cs}\right.$ and $\left.{ }^{134} \mathrm{Cs}\right)$ of ewes and lambs $(n=6)$ were assessed by whole-body counting ; the milk intake of the lambs was estimated as a function of their daily weight increase. The mean milk and meat transfers in ewes, between D10 and D30 after lambing were respectively $4.7 \pm 1.4 \%$ and $7.9 \pm$ $1.7 \%$. At birth the mean caesium body burden of the lambs was $3.2 \pm 8 \%$ of the dam body burden ; at weaning (D30) the mean retention of the ingested radiocaesium was $39 \pm 6 \%$ vs $27.3 \pm 3.2 \%$ for the dams. The spontaneous decorporation half-time of caesium in lambs at weaning was about 14.5 days (monoexponential curve) vs about 26 days for the dams (long component of bi-exponential curve). Meat transfers, uptake and spontaneous decorporation parameters were also studied : 1) in 4 lambs from 1 to 3 months after weaning ; and 2) in 3 young animals between 9 and 10 months after birth. The values of the different parameters of caesium transit in neonates and lambs are discussed with regard to contamination accident management.

\footnotetext{
* Laboratoire de radiobiologie appliquée, Département de pathologie et de toxicologie expérimentales, Direction des sciences du vivant, Commissariat à l'énergie atomique, 91191 Gif-sur-Yvette cedex, France.
} 


\section{Introduction}

II n'existe jusqu'ici dans la littérature que peu de données sur le transfert aux foetus du radiocésium ingéré chez les brebis gestantes, aux nouveaux-nés à partir du lait maternel pendant l'allaitement et aux jeunes agneaux après le sevrage. Pourtant, les valeurs habituellement élevées de l'absorption de nombreux minéraux chez le jeune peuvent poser un problème après une contamination des aliments du bétail et des fourrages par les retombées d'un accident nucléaire. Certains auteurs ont montré que, pour le césium, le transfert au fœtus était assez limité chez les ovins $[3,14]$ mais que le transfert au muscle à partir du lait ingéré était, au contraire, très élevé et égal à 4 à 8 fois la valeur observée chez les adultes à partir du fourrage $[8-9,14]$.

Le travail décrit ci-dessous rassemble plusieurs expérimentations de contamination orale, conduites sur différents groupes d'animaux à différents âges, et concerne principalement le suivi de la contamination des mères et de leurs produits avant et après la mise bas. D'autre part, il donne des valeurs de coefficients de transfert et de vitesse de l'élimination spontanée chez le jeune après le sevrage et jusqu'à l'âge adulte.

\section{Matériel et méthodes}

\section{Animaux}

a) Brebis gestantes: quatre brebis adultes, âgées de 4 et 5 ans, croisées Frisonne-Sarde-Lacaune, ont été utilisées : deux au printemps 1990 , B1 et B2, ont reçu du foin contaminé 40 jours avant et 30 jours après la mise bas ; deux au printemps 1991, B3 et B4, ont reçu le même foin 30 jours avant et 30 jours après la mise bas. Ce foin avait été récolté dans le sud-est de la France en juin 1986 et contenait, en 1990, environ $3500 \mathrm{~Bq} / \mathrm{kg}$ de matière sèche (MS) de ${ }^{137} \mathrm{Cs}$ et $500 \mathrm{~Bq} / \mathrm{kg}-\mathrm{MS}$ de ${ }^{134} \mathrm{Cs}$. La teneur en ${ }^{40} \mathrm{~K}$ était, en moyenne, de $950 \mathrm{~Bq} / \mathrm{kg}-\mathrm{MS}$ soit environ $30 \mathrm{~g} / \mathrm{kg}-\mathrm{MS}$ de potassium stable.

b) Agneaux de lait : les quatre brebis ont eu chacune 2 agneaux :

- dix jours après sa naissance, l'agneau L4 a été écarté de sa mère qui a présenté à cette date une mammite unilatérale; il a été nourri au lait artificiel jusqu'au sevrage, 20 jours plus tard;

- l'agneau L2 est mort à la naissance, soit 39 jours après le début de la contamination de sa mère ;

- bien que la mise bas ait eu lieu pour les 2 agneaux 29 jours après le début de la contamination de la brebis B4, l'agneau L8 est probablement mort vers $\mathrm{J} 24-\mathrm{J} 25$, car sa mère a présenté à cette date une anorexie qui a duré jusqu'après la mise bas.

c) Agneaux sevrés : deux autres groupes de 2 agneaux (L9-L10 et L11-L12) ont reçu du foin contaminé (et des aliments concentrés) de J50 
à J65 après la naissance ; chaque groupe était laissé dans la même cage ; la charge en radiocésium était évaluée par comptage global ; l'élimination spontanée a été suivie de $\mathrm{J} 66$ à $\mathrm{J} 84$ pour chaque animal ; une nouvelle expérience de contamination a été entreprise avec le même foin contaminé de $\mathrm{J} 85$ à $\mathrm{J} 100$; dans ce dernier cas, seule la période de charge et la rétention à $\mathrm{J} 100$ ont été suivies.

d) Jeunes adultes : les agneaux $\mathrm{L} 1, \mathrm{~L} 3$ et $\mathrm{L} 4$ ont été réutilisés à l'âge de 9-10 mois (La1, La3 et La4) pour une courte période de contamination de 15 jours et un suivi de l'élimination spontanée pendant 18 jours.

Les caractéristiques des animaux sont regroupées dans le tableau I.

TABLEAU 1

Caractéristiques des animaux.

Animal characteristics

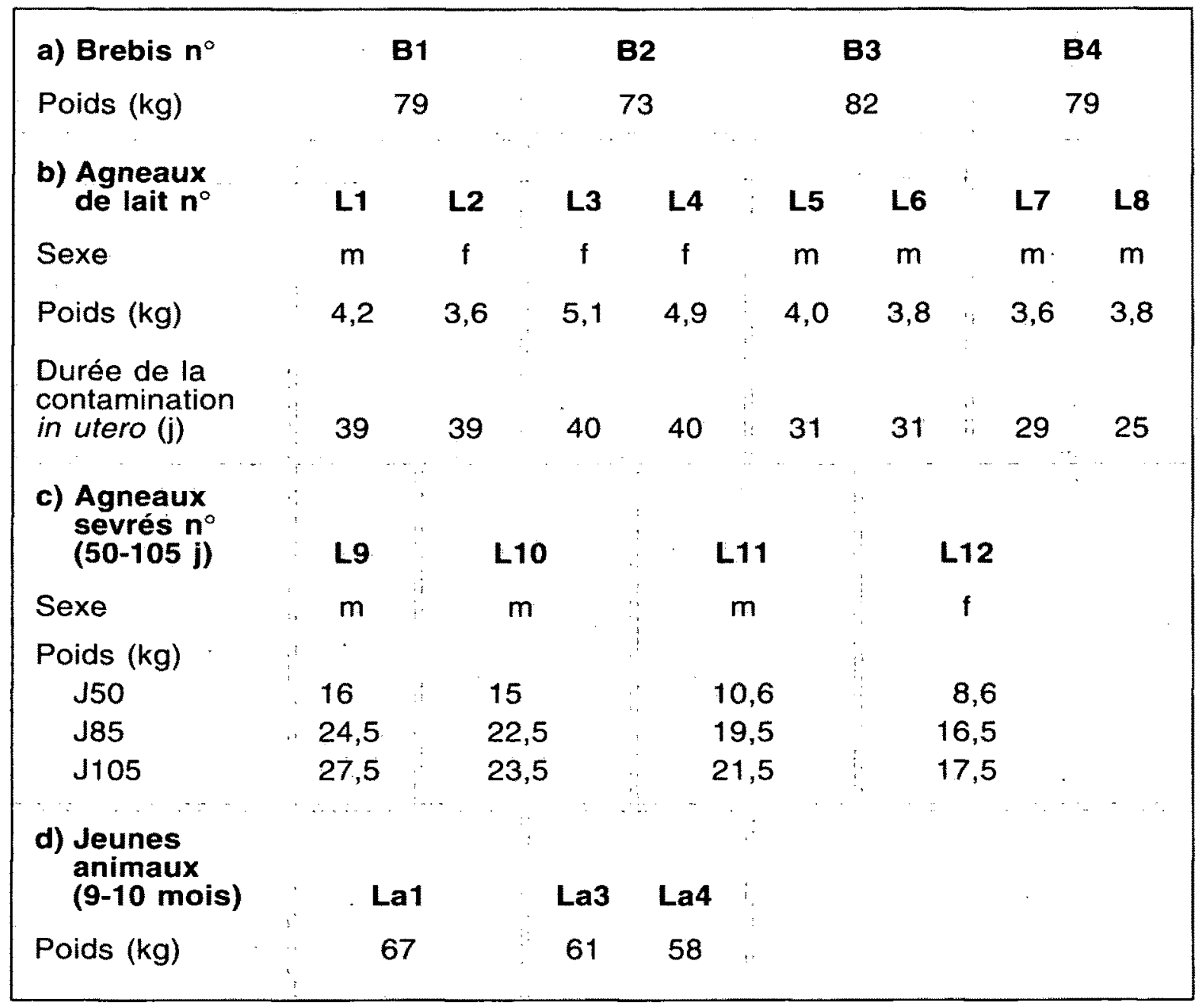




\section{Alimentation}

Le foin se présentait sous la forme de balles de $15-18 \mathrm{~kg}$. Ces balles étaient mesurées par comptage global dans l'installation destinée aux animaux décrite ci-dessous. L'étalonnage a été réalisé en prenant de nombreux échantillons, minéralisés puis mesurés, sur une dizaine de balles de foin de radioactivité différente ; le rendement de comptage était dans ces conditions de $0,18 \mathrm{cpm} / \mathrm{Bq}$ sur les pics de ${ }^{137} \mathrm{Cs}$ et ${ }^{134} \mathrm{Cs}$.

La consommation des animaux adultes étant de 1 à $2 \mathrm{~kg}$ par jour selon les sujets, les balles étaient regroupées selon leur niveau de radioactivité pour nourrir un même animal pendant la durée de l'expérimentation et, ainsi, essayer d'assurer un taux de contamination journalière constant. Les apports quotidiens étaient pesés, les refus pesés et mesurés lorsque chaque balle de foin était terminée. Nous avons dû nous résoudre à chauffer les balles pendant $18 \mathrm{~h}$ à $80^{\circ} \mathrm{C}$ pour les rendre plus appétentes et ôter l'odeur de moisi que présentaient certaines d'entre elles ; la matière sèche (MS) a été déterminée après cette phase de chauffage.

Les brebis $\mathrm{B} 1$ et $\mathrm{B} 2$ ont reçu, en plus de leur ration de foin, un complément de pulpe $(600 \mathrm{~g} / \mathrm{j})$ et de grains $(600 \mathrm{~g} / \mathrm{j})$; les brebis B3 et B4 ont été relativement rationnées $(300-400 \mathrm{~g} / \mathrm{j}$ d'aliments concentrés) pour essayer d'accroître leur consommation de foin contaminé. II est certain que ce dernier groupe a reçu une alimentation insuffisante pour assurer un taux élevé de production laitière et, corrélativement, une bonne croissance des agneaux (tab. I).

Les agneaux plus âgés, après le sevrage, recevaient du foin contaminé à volonté avec un complément minimal d'aliments concentrés.

Pendant la période de décontamination, qui correspondait à celle du tarissement chez les brebis et du sevrage chez les jeunes, les animaux recevaient à volonté du foin de bonne qualité non contaminé.

La consommation de lait des agneaux laissés près de leur mère était calculée à l'aide de l'abaque fournie par Jarrige [10] en utilisant la prise de poids journalière : il existe, jusqu'au sevrage, une relation linéaire entre le taux de croissance des jeunes et la production laitière de la mère. La radioactivité spécifique du lait était déterminée sur des échantillons de $10 \mathrm{ml}$ prélevés quotidiennement à la mamelle.

Les urines et les fèces étaient prélevées, pesées et mesurées tous les deux jours, pour établir les taux d'absorption et de rétention.

\section{Définition des différents coefficients}

- Les coefficients de transfert de la contamination apportée par la nourriture au lait et à la viande sont définis comme suit : 


$$
\begin{gathered}
F m(\text { lait })=\frac{\text { radioactivité } \mathrm{d}^{\prime} 1 \mathrm{~kg} \text { de lait }}{\text { quantité de radioactivité ingérée quotidiennement }} \\
F f(\text { viande })=\frac{\text { radioactivité } \mathrm{d}^{\prime} 1 \mathrm{~kg} \text { de viande }}{\text { quantité de radioactivité ingérée quotidiennement }}
\end{gathered}
$$

- Les coefficients d'absorption et de rétention ont été établis selon les formules classiques utilisées en nutrition :

$$
\text { Coefficient d'absorption }=\frac{\text { césium ingéré }- \text { césium fécal }}{\text { césium ingéré }}
$$

Coef. de rétention $=\frac{\text { césium ingéré }-(\text { césium fécal }+ \text { césium urinaire })}{\text { césium ingéré }}$

\section{Comptages globaux}

La radioactivité globale des animaux était mesurée dans une enceinte blindée $(2,5 \times 2 \times 2,2 \mathrm{~m})$ équipée de 4 détecteurs opposés par paires (cristaux Nal, $102 \times 102 \mathrm{~mm}$ ) ; dans la bande de mesure entre 500 et $900 \mathrm{keV}$ le mouvement propre était de $500 \mathrm{cpm}$. Les méthodes d'étalonnage et de comptage ont été décrites précédemment [7] ; rappelons seulement que les particularités anatomiques des ruminants conduisent à effectuer une double mesure, avec et sans protection latérale en plomb, pour évaluer séparément la charge du muscle et celle du rumen. Pour les brebis, les mesures avaient lieu tous les jours pendant les périodes de charge et de décharge, et 2-3 fois par semaine pendant la phase de quasi-équilibre. La mesure des agneaux a nécessité l'emploi de plusieurs types de cage de contention en bois et de plusieurs facteurs d'étalonnage établis sur des fantômes de bidons de plastique ; les animaux étaient mesurés tous les 2 jours. Le rendement de comptage dans la bande $500-900 \mathrm{keV}$ était de $0,6 \mathrm{cpm} / \mathrm{Bq}$ pour les brebis et $0,8 \mathrm{cpm} / \mathrm{Bq}$ pour les agneaux.

La mesure de la radioactivité des différents organes des deux agneaux morts-nés a été réalisée après minéralisation des prélèvements.

\section{Traitement des données}

Les courbes de charge et d'élimination spontanée ont été ajustées à des modèles linéaires, mono ou bi-exponentiels à l'aide du programme de calcul Asystant (N.D.) de la société Asyst Software (New York, USA). 


\section{Résultats}

1. Les coefficients de transfert (Ff) pour la viande ont été déterminés chez les brebis pendant la phase de quasi-équilibre, entre le vingtième et le trentième jour après la mise bas. Un échantillon de muscle (4 et $7 \mathrm{~g}$ ) a été prélevé chirurgicalement dans la région du cou chez les brebis B3 et B4 ; les valeurs déterminées par comptage global ont été utilisées pour les brebis $\mathrm{B} 1$ et $\mathrm{B} 2$, après soustraction de la charge du rumen. II existe, en effet, une relation entre le coefficient de transfert $F f$ mesuré sur des échantillons de muscle et la charge corporelle mesurée par comptage global et exprimée en pourcentage de la quantité ingérée quotidiennement $(Q)$ :

$$
F f=2,52 \times Q-2,66 \quad(r=0,93, n=10)
$$

Au début de la contamination orale, $F f=0$ et la charge des animaux (105\% de la quantité ingérée) correspond à la charge du rumen [7]. Dans la viande, les coefficients de transfert $F f$ ont été respectivement 8,45 et 6,1 pour $\mathrm{B} 1$ et $\mathrm{B} 2$, et 9,45 et 9 pour $\mathrm{B} 3$ et $\mathrm{B} 4$.

La charge des agneaux à la naissance est présentée dans le tableau II en fonction de la durée de la contamination in utero et de la quantité de radiocésium ingérée quotidiennement par la mère. Les pourcentages transférés aux fœtus sont très comparables pour les différents animaux ( $m=15,6 \pm 1$ ) sauf pour le fœtus mort in utero probablement 4-5 jours avant le part pour lequel le pourcentage est d'environ la moitié. La répartition du radiocésium chez les agneaux morts-nés est donnée au tableau III.

TABLEAU $\|$

Charge en radiocésium des agneaux à la naissance en fonction de la durée de la contamination et de la charge de la mère. Radiocaesium body burdens of newborn lambs vs contamination durations and body burdens of the dams

\begin{tabular}{|c|c|c|c|c|c|c|c|c|}
\hline Agneau $n^{\circ}$ & L1 & L2 $^{*}$ & $\mathrm{~L} 3$ & L4 & L5 & L6 & $L 7$ & $\mathrm{LB}^{*}$ \\
\hline $\begin{array}{l}\text { Charge en césium } \\
\text { à la naissance }(\mathrm{Bq} / \mathrm{kg})\end{array}$ & 142 & 137 & 134 & 128 & 203 & 197 & 134 & 62 \\
\hline $\begin{array}{l}Q \text { de césium ingérée } \\
\text { par la mère }(\mathrm{Bq} / \mathrm{j})\end{array}$ & \multicolumn{4}{|c|}{$(J 0-40)$} & \multicolumn{2}{|c|}{5038} & 2965 & \\
\hline $\begin{array}{l}\text { Durée de la contamina- } \\
\text { tion avant le part }(\mathrm{Bq} / \mathrm{j})\end{array}$ & \multicolumn{2}{|c|}{39} & \multicolumn{2}{|c|}{40} & \multicolumn{2}{|c|}{31} & 29 & 25 \\
\hline $\begin{array}{l}\text { Transfert aux agneaux } \\
\text { à la naissance en \% } \\
\text { de l'ingéré quotidien }\end{array}$ & 17,1 & 15,3 & 15,2 & 14 & 16,3 & 14,9 & 16,5 & 8,1 \\
\hline Transfert/kg & 4,1 & 3,9 & 3 & 2,8 & 4,1 & 3,9 & 4,6 & 2,1 \\
\hline
\end{tabular}

* Agneaux morts nés. 
TABLEAU III

Distribution du radiocésium dans les carcasses des agneaux morts-nés. Radiocaesium distribution in stillborn lamb carcasses

\begin{tabular}{|c|c|c|c|c|c|c|c|c|}
\hline meste & pois & (g) & $\mathrm{Bq}$ & aux & B & & $\% \mathrm{BO}$ & otaux \\
\hline+2 & $\mathbf{L 2}$ & L8 8 & L2 & L8 & $L 2$ & $\mathbf{L}$ & L2 & L8 \\
\hline Corps entier & 3900 & 3800 & 534 & 235 & 137 & 62 & 100 & 100 \\
\hline Rein & 50 & 20 & 9 & 1,3 & 180 & 63 & 1,7 & 0,5 \\
\hline Tractus digestif & 350 & 208 & 49 & 27 & 140 & 128 & 9 & 11 \\
\hline Foie & 95 & 90 & 15 & 16 & 160 & 178 & 2,8 & 6,8 \\
\hline Poumon & 110 & 104 & 15 & 4 & 140 & 38 & 2,8 & 1,7 \\
\hline Cœur & 40 & 32 & 7 & 5 & 170 & 153 & 1,3 & 2,1 \\
\hline Muscle* (total) & 1170 & 1140 & 374 & 175 & 320 & 154 & 70 & 75 \\
\hline
\end{tabular}

* Le pourcentage du poids total de muscle a été estimé à $30 \%$ du poids total de la carcasse, d'après Mihardja [11].

Après la mise bas $(\mathrm{J} 0)$ la rétention des agneaux contaminés par le lait maternel est en moyenne $51,5 \pm 18 \%(n=6)$ entre J0 et $\mathrm{J} 10$, et $36,2 \pm$ $5 \%(n=5)$ entre $\mathrm{J} 11$ et J30, date du sevrage (tab. IV).

Les coefficients de transfert apparents (car les animaux ne sont pas à l'équilibre) pour le muscle des agneaux au moment du sevrage sont compris entre 0,76 et $1,40(m=1,1 \pm 0,37)$ en admettant que l'espace césium est égal à cet âge à $75 \%$ du poids vif [1]

Les coefficients de transfert pour le lait Fm ont été établis chez les brebis entre $\mathrm{J} 10$ et $\mathrm{J} 30$ après le part : $F m=3,7$ et $4,45 \%$ pour les brebis $\mathrm{B} 1$ et $\mathrm{B} 2$, et $F m=4,35$ et $7,1 \%$ pour les brebis B3 et B4 (tab. III).

Enfin, un point important doit être souligné pour justifier les comparaisons avec les expérimentations antérieures de Cousi, et Daburon et al. $[5,7]$ : nous n'avons pas trouvé de différences significatives entre les coefficients d'absorption et de rétention chez les animaux nourris avec du foin séché naturellement en 1987-88, respectivement $40,2 \pm 3,4$ et $27,3 \pm 3$, et ceux qui ont reçu en 1990-91 du foin chauffé à $80^{\circ} \mathrm{C}$ pendant $18 \mathrm{~h}$, respectivement $39,4 \pm 4,5$ et $25+4$. 
TABLEAU IV

Transfert du radiocésium au lait et à la viande des brebis et rétention par les agneaux. Radiocaesium transfer to ewes' milk and meat and retention by lambs

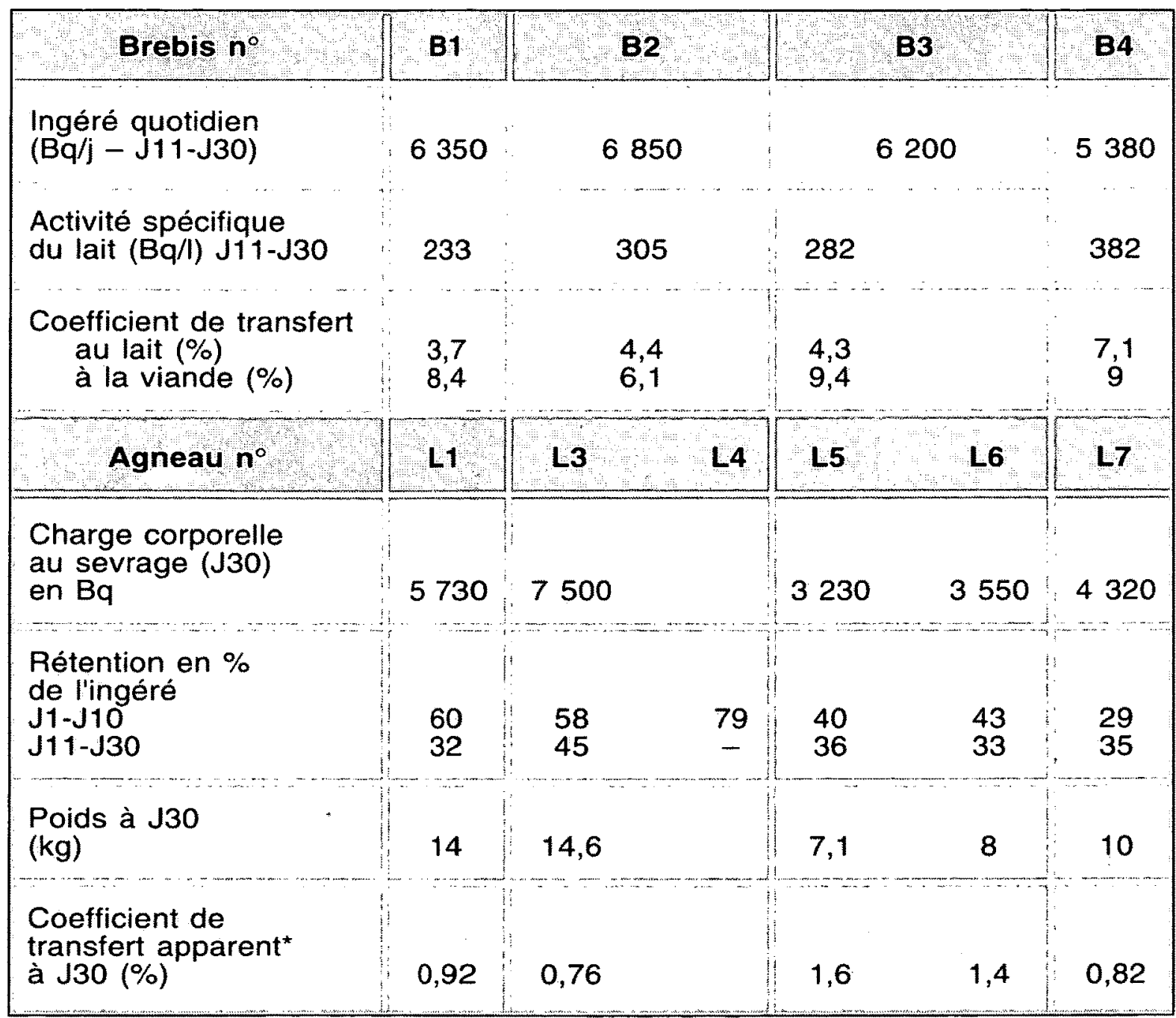

* L'espace césium est estimé à $75 \%$ du poids corporel [1] et la radioactivité de $1 \mathrm{~kg}$ de muscle a été calculée en divisant la charge corporelle par $75 \%$ du poids.

2. Incorporation et élimination spontanée du radiocésium chez les brebis et les agneaux (fig. 1, courbes 1, 2, 3 et 4).

La charge des brebis adultes, évaluée par comptage global, peut être ajustée à une équation simplifiée, ne prenant pas en compte l'élimination spontanée, de la forme:

$$
Y=A(1-\exp -k t) \text { avec } k=\operatorname{Ln} 2 / T,
$$

$A$ étant la valeur de la charge à la période de quasi-équilibre (1 mois), $T$ étant la période apparente de charge.

Pour les quatre brebis gestantes (B1, B2, B3 et B4), la période moyenne de charge était de 12,2 jours, avec des valeurs extrêmes de 9,4 et 15,8 ; comme pour les valeurs des périodes données plus loin, 
cette moyenne n'est donnée qu'à titre indicatif, car elle n'a évidemment pas de valeur statistique.

Entre J0 et J30 le taux de croissance pondérale et l'augmentation de la charge corporelle en radiocésium chez les agneaux de la naissance au sevrage peuvent être ajustés à une équation linéaire avec des pentes en moyenne tout à fait semblables et égales à 3,9\% par jour pour les deux paramètres.

Les courbes d'élimination spontanée (tab. V) chez les brebis après l'arrêt de la contamination orale ont été ajustées à la somme de 2 exponentielles $[4,7]$; la valeur moyenne de la période rapide était de 2,6 j, avec des limites de 1,8-3,3 j, celle de la période longue $26,3 \mathrm{j}$, avec des limites de 21,5-29,8 j. Pour les agneaux, juste après le sevrage et au moment de l'arrêt de la contamination orale, le meilleur ajustement a été obtenu avec une courbe mono-exponentielle de période moyenne $14,5 j$ et des limites de 13,1-16,1 j. Pour les agneaux un peu plus âgés (65 j)
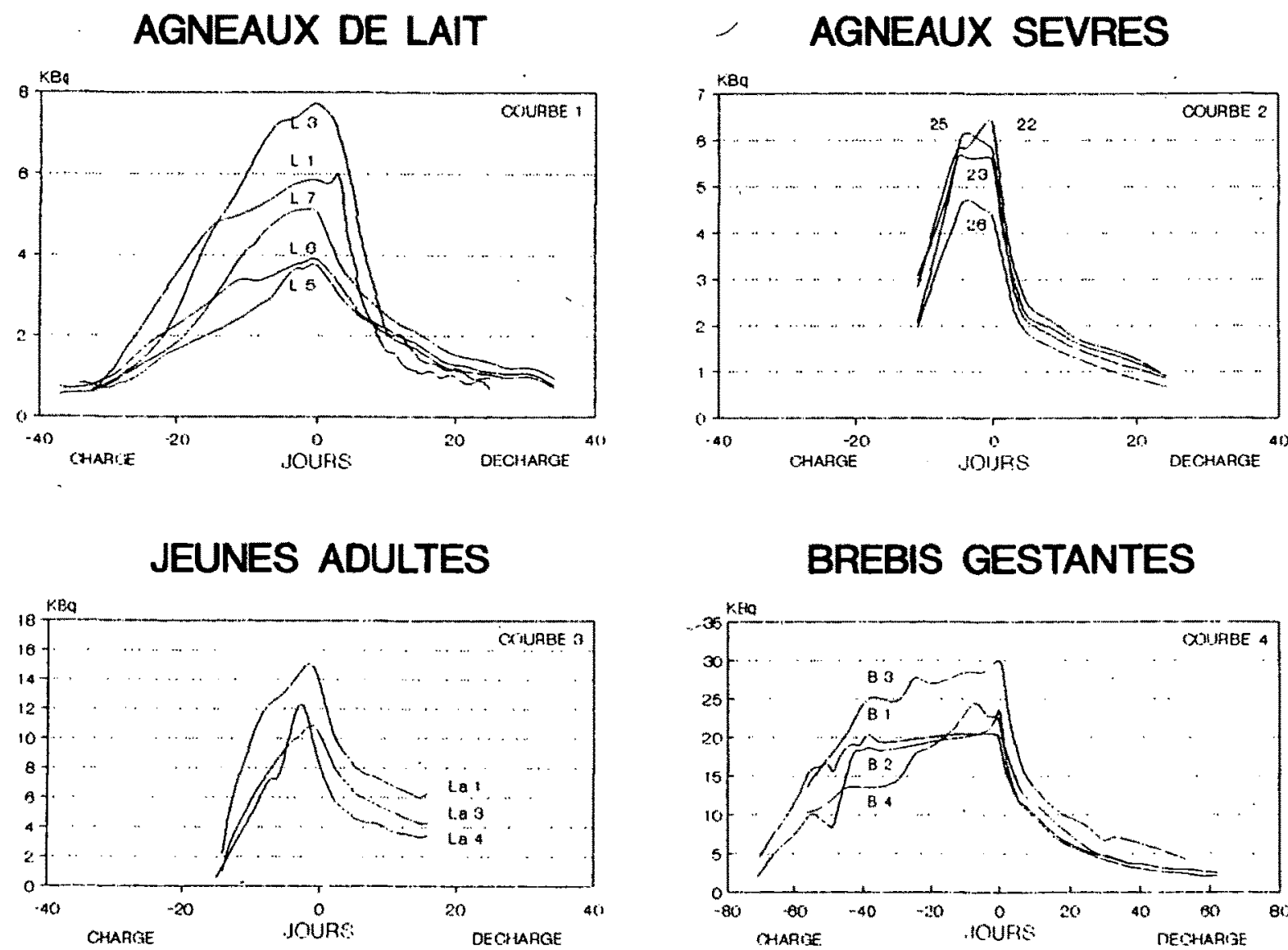

Fig. 1. - Courbes représentatives de la charge et de la décorporation spontanée des différents groupes d'animaux étudiés.

Representative body burden and spontaneous decorporation curves for the various groups of animals studied: (1) suckling lambs, 2) weaned lambs, 3) young adults, 4) pregnant ewes). 
TABLEAU $V$

Comparaison des paramètres d'incorporation et d'élimination spontanée du radiocésium dans les différents groupes d'animaux.

Compared caesium intake and spontaneous excretion parameters in the various groups

\begin{tabular}{|c|c|c|c|c|c|c|}
\hline Animaux & $\begin{array}{l}\text { Période } \\
\text { de charge } \\
(\mathbf{0})\end{array}$ & $\begin{array}{l}\text { Coefficient } \\
\text { dabsorp } \\
\text { tion }(\%)\end{array}$ & $\begin{array}{l}\text { Coefficient } \\
\text { de réten } \\
\text { tion }(\%)\end{array}$ & $\begin{array}{l}\text { Transfert } \\
\text { aut muscle } \\
(\%)\end{array}$ & \multicolumn{2}{|c|}{$\begin{array}{l}\text { Periodede } \\
\text { décharge (i) }\end{array}$} \\
\hline $\begin{array}{l}\text { Agneaux } \\
\text { de lait }(n=5)\end{array}$ & $3,9 \% / j$ & - & $36 \pm 5$ & $160 \pm 40$ & $(13)^{1}$ & $\begin{array}{l}4,5 \\
-16,1)\end{array}$ \\
\hline $\begin{array}{l}\text { Agneaux sevrés } \\
\text { J50-J65 } \\
(n=2 \times 2)\end{array}$ & $\begin{array}{r}5,2 \\
3,9-5,1\end{array}$ & $35-46$ & $22-30$ & $22 \cdot 33$ & $\begin{array}{c}1,2 \\
(1,1-1,3)\end{array}$ & $\begin{array}{c}14,1 \\
(12,8-15,5)\end{array}$ \\
\hline J85-J100 & - & $49-59$ & $29-34$ & $21-30$ & - & - \\
\hline $\begin{array}{l}\text { Jeunes adultes } \\
\text { ( } 9-10 \text { mois) } \\
\text { brebis } \\
(n=2)\end{array}$ & $10-11$ & $45-57$ & $28-32$ & $14-17$ & $\begin{array}{c}0,9 \\
(0,8-1)\end{array}$ & $\begin{array}{c}18,8 \\
(17,3-20,4)\end{array}$ \\
\hline Bélier & 5,4 & 42 & 24 & 12 & 0,8 & 23 \\
\hline $\begin{array}{l}\text { Brebis sèches } \\
(3-5 \text { ans }) \\
(n=4)[6]\end{array}$ & $\begin{array}{c}7 \\
(5,8-8,5)\end{array}$ & $40,2 \pm 3,4$ & $27 \pm 3$ & $11 \pm 1,3$ & $\begin{array}{c}1,8 \\
(1-2,2)\end{array}$ & $\begin{array}{c}26,5 \\
(24,1-28,9)\end{array}$ \\
\hline $\begin{array}{l}\text { Brebis gestantes } \\
\text { ( } 4-5 \text { ans) } n=4\end{array}$ & $\begin{array}{c}12,2 \\
9,4-15,8\end{array}$ & $39,4 \pm 4,5$ & $25 \pm 4$ & $8,2 \pm 1,5$ & $\begin{array}{c}2,6 \\
(1,8-3,3)\end{array}$ & $\begin{array}{c}26,3 \\
(21,5-29,8)\end{array}$ \\
\hline
\end{tabular}

NB : Le chiffre supérieur donné pour les valeurs des périodes de charge et de décorporation correspond à la moyenne des valeurs du groupe d'animaux; les chiffres donnés à la ligne inférieure correspondent aux valeurs extrêmes. Les numéros des groupes d'animaux renvoient à ceux des courbes de la figure 1 .

on retrouve un modèle bi-exponentiel avec des valeurs des périodes rapides et lentes égales respectivement à $1,2 \mathrm{j}$ (limites $1,1-1,3$ ) et $14,1 \mathrm{j}$ (limites 12,8-15,5). Pour les jeunes adultes (9-10 mois) la période courte reste autour de 1 j (limites $0,8-1$ ) mais la période longue s'allonge à plus de $20 \mathrm{j}$ (limites 17,3-23). Le tableau $V$ regroupe les valeurs obtenues dans les différents lots d'animaux.

\section{Discussion}

Le but de cette étude était, d'abord, de tester la faisabilité d'une expérimentation de contamination orale chez des brebis gestantes placées en cage à métabolisme avant la mise bas et laissées avec leurs produits jusqu'au sevrage, 30 jours après le part. Les bilans sont faciles à réaliser sur les brebis isolées ; après la mise bas, une petite contamination des excreta apparaît avec les urines et les fèces des agneaux. La quantité de lait produite journellement semble correctement appréciée par la prise de 
poids des agneaux ; pendant la dernière semaine de cohabitation entre mère et produits, les agneaux commencent à consommer, en plus du lait, de petites quantités de foin contaminé : les modifications entraînées dans les bilans ne semblent pas très importantes car les courbes de croissance et de charge en radiocésium restent parallèles.

Nous pouvons comparer les coefficients de transfert établis tant pour le lait que pour la viande, au cours d'expérimentations antérieures, avec le même foin, utilisé précocément après la récolte en 1987-1988 [5,7] avec ceux définis au cours de cette expérimentation en 1990-1991. Les valeurs moyennes passent respectivement de $7,5 \pm 0,5 \%(n=3)$ à 4,9 $\pm 1,5 \%$ pour le lait et de $11 \pm 1,3 \%(n=5)$ à $8,2 \pm 1,5 \%$ pour la viande. Cette baisse de 1/3 environ pour le lait et de 1/4 pour la viande n'est pas significative (test de Mann-Whitney). Bien que la qualité du foin se soit beaucoup dégradée entre les périodes 1987-88 et 1990-91, les valeurs des coefficients d'absorption et de rétention à partir de ce même foin contaminé ne diffèrent pas significativement entre les deux séries d'expérimentations (tab. V).

A la naissance, c'est-à-dire après 30 à 40 jours de contamination, le taux de contamination des agneaux est égal à $3,2 \pm 0,8 \%(n=6)$ de la charge de la mère évaluée par comptage global. Buldakov [3] avait déjà noté la faiblesse de cette accumulation en donnant des valeurs comprises entre 1,5 et $2 \%$; mais nous avons observé un taux de contamination par kilogramme de $60 \%$ de celui de la mère contre seulement $10 \%$ par Buldakov. Chez les sujets morts-nés la radioactivité spécifique des différents organes semblait assez homogène avec une valeur plus élevée dans le muscle observée également par Buldakov [3].

En comparant la distribution du césium donnée par Bonsembiante et al. [2] pour les moutons ingérant pendant 129 jours du foin contaminé, on observe une concentration moyenne dans les reins, le tube digestif, le foie, le poumon et le cœur environ deux fois plus élevée que chez les deux fœtus $L 2$ et $L 8$; on sait depuis longtemps que la fixation du césium dans les muscles dépend de leur activité métabolique. La charge totale du fœetus $L 7$ est le double de celle de son frère L8 mort probablement 45 jours plut tôt ; d'autre part, les répartitions en pourcentage dans les organes sont assez semblables entre les deux morts-nés L2 et L8. On pourrait donc supposer que les derniers jours de gestation voient augmenter considérablement la fixation de radiocésium par le fœetus, peutêtre avec la maturation du tissu musculaire ; mais nous avons vu précédemment que le pourcentage du césium ingéré transféré au fœetus était le même après 30 ou 40 jours de contamination de la mère.

Howard et al. [8-9] ont donné des valeurs de coefficients de transfert à la viande proches de nos résultats [7] (12\% contre $11 \%)$; pour les agneaux de lait, le même auteur donne pour coefficient de transfert à la viande la valeur de $120 \%$ contre $160 \%$ pour la moyenne de nos animaux le jour du sevrage (tab. IV). 
Les coefficients d'absorption et de rétention semblent diminuer avec l'âge des animaux jusqu'à une valeur moyenne de respectivement 40 et $27 \%$ chez les brebis adultes. Vandecasteele et al. [14] avaient également noté chez des sujets adultes une valeur moyenne de $26 \pm 6 \%$.

La vitesse de l'élimination spontanée du radiocésium semble inversement proportionnelle à l'âge. Elle varie chez nos animaux de 14,5 j en moyenne à l'âge de 30 jours. Vandecasteele et al. [14] avaient trouvé 10$11 \mathrm{j}$, à environ $26 \mathrm{j}$ pour la composante lente. Enfin, les différences observées dans les courbes de charge des brebis pleines et vides (environ $12 \mathrm{j}$ contre 7) ne se retrouvent pas pour les courbes d'élimination spontanée.

\section{Conclusion}

Nous avons voulu apporter dans ce travail de nombreuses données qui ne sont généralement pas regroupées dans la littérature. Elles devraient permettre une comparaison plus facile des différents paramètres de l'incorporation et de l'élimination spontanée du radiocésium ingéré chez les ovins à différents âges et selon différents états physiologiques (lactation, gestation).

En ce qui concerne l'attitude à avoir en cas d'accident de contamination, il faut remarquer que, si les jeunes animaux se chargent rapidement, leur élimination spontanée est également rapide ; le faible transfert du césium ingéré par la mère au fœus doit être également souligné. II semble donc plus pragmatique et moins coûteux de traiter le troupeau en entier avec des additifs bloquant l'absorption digestive du césium et laisser les animaux se décontaminer naturellement plutôt que d'entreprendre une alimentation de substitution avec des laits artificiels. Nous avons observé précédemment [7] que si les substances complexant le césium dans le tube digestif étaient incapables d'accélérer son élimination spontanée chez les animaux, elles semblent susceptibles d'accélérer la décroissance de la radioactivité spécifique du lait. D'autres études sont en cours pour confirmer cette observation.

\section{Remerciement}

Nous devons remercier ici les techniciens animaliers du LRA qui ont assuré les soins aux animaux et la plupart des prélèvements nécessaires aux expérimentations : MM. J.J. Leplat, J.F. Dossin et Y. Mellerin. F. Aymard (stagiaire CEA) a participé très activement à l'expérimentation menée en 1991. Y. Tricaud a contribué à la mise en forme des résultats et a assuré leur représentation graphique. 


\section{RÉFÉRENCES}

[1] AGASSE J.C. - Contribution à l'étude des secteurs hydriques du mouton à l'aide des radioéléments. Thèse vétérinaire, Université Paul Sabatier, Toulouse, 1971.

[2] BONSEMBIANTE M., ANDRIGHETTO I., RAMANZIN M., MOSCHINI G. Observation on transfer of caesium to meat and milk in sheep and goat. 42th Annual meeting of the European association of animal production, Berlin, Germany, 8-12 Sept. 1991.

[3] BULDAKOV L.A. - Biological effects of radioactive isotopes. Metabolism and biological effects of caesium 137 in sheep. In : Distribution, biological effects and accelerated excretion of radioactive isotopes (Yu. I. Moskalev, Ed.) 1964. AEC Translation, AEC-TR-7590, Springfield : NTIS, 1974, 178-193.

[4] COUGHTREY P.J., THORNE M.C. - Radionuclide distribution and transport in terrestrial and aquatic ecosystems. Rotterdam : A.A. Balkema, 1983, Vol. 1, 374-377.

[5] COUSI J. - Etude expérimentale de la contamination des ovins par les radiocaesium 134 et 137. Thèse vétérinaire, Faculté Médecine, Créteil, 1989.

[6] DABURON F., ARCHIMBAUD Y., COUSI J., FAYART G. - Radiocaesium and radioidine contamination in ewes, countermeasures. In : Environmental contamination following a major nuclear accident, Vienna, October 16-20, 1989. Vienne : AIEA, 1990, 155-156.

[7] DABURON F., ARCHIMBAUD Y., COUSI J., FAYART G., HOFFSCHIR D., CHEVALLEREAU I., LE CREFF H., GUEGUEN L. - Radiocaesium transfer to ewes fed contaminated hay after the Chernobyl accident : effect of vermiculite and AFCF (ammonium ferricyanoferrate) as countermeasures. J. Environ. Radioact., 1991, 14, 73-84.

[8] HOWARD B.J. - A comparison of radiocaesium transfer coefficients for sheep milk and muscle derived from both field and laboratory studies. Sci. Total Environ., 1989, 85, 189-198.

[9] HOWARD B.J., MAYES R.W., BERESFORD N.A., LAMB C.S. - Transfer of radiocaesium from different environmental sources to ewes and suckling lambs. Health Phys., 1989, 57 (4), 579-586.

[10] JARRIGE R. - Principes de la nutrition et de l'alimentation des ruminants. Paris : Institut national de la recherche agronomique, 1978, p. 408.

[11] MIHARDJA S. - Contribution à l'étude de la composition corporelle d'agneaux mâles en croissance appartenant à 5 génotypes. Thèse d'Université, Université des sciences et techniques du Languedoc, Montpellier, 1981.

[12] MOSS B.W., UNSWORTH E.F., MCMURRAY C.H., PEARCE J., KILPATRICK D.J. - Studies on the uptake, partition and retention of ionic and fallout radiocaesium by suckling and weaned lambs. Sci. Total Environ., 1989, 85, 91-106.

[13] NG Y.C., COLSHER C.S., THOMSON S.E. - Transfer factors for assessing the dose from radionuclides in agricultural products. In "Biological implications of radionuclides released from nuclear industries", Vienna, March 26-30, 1979. Vienna: AIEA, 1979, Vol. 2, 295-318.

[14] VANDECASTEELE C.M., VAN HEES M., CULOT J.P., VANKERKOM J. Radiocaesium metabolism in pregnant ewes and their progeny. Sci. Total Environ., $1989,85,213-223$. 